\title{
Lack of funds for technology "could undermine' Mars mission science
}

Washington. An ambitious US plan to launch spacecraft to Mars every two years could be hampered by a lack of funding for new technology, according to both a National Research Council report and scientists and contractors familiar with the proposed missions.

Unless NASA invests more money in miniaturized scientific instruments, large roving vehicles and other key technologies, the proposed Mars missions may have their scientific objectives "seriously undermined", according to a report to be released this week by the Committee on Planetary and Lunar Exploration (COMPLEX).

The committee, chaired by Ronald Greeley of Arizona State University, is particularly concerned about funding for scientific 'microinstruments', which it says has not kept up with spacecraft design.

The panel also says that answering key questions about Mars - particularly conducting a comprehensive search for existing or fossil life - will require larger and more capable landers and roving vehicles than those NASA now has in mind. 'Minirovers' of the type that the Mars Pathfinder spacecraft will land on the planet's surface next July have a limited range, measured in tens of metres, and can last only a few weeks.

The goals and design of NASA's Mars Surveyor programme, which will spend about $\$ 1$ billion over the next decade to visit Mars at each 26-month opportunity, have wide support among space scientists. But many say they are concerned that the programme is underfunded.

Daniel Goldin, the administrator of NASA, only added to such concerns by challenging his engineers, at a recent symposium to mark the 20th anniversary of Viking's landing on Mars, to return a sample of the planet as early as 2003. A sample return was not part of the original Mars Surveyor plan. Most observers, inside and outside NASA, doubt whether it can be accommodated within the existing budget.

"Money is the key to this programme," says Donna Shirley, Mars exploration manager at the Jet Propulsion Laboratory (JPL). Shirley boasts in her public presentations that the $\$ 150$ million price of each Surveyor mission is about the same as that of a Hollywood blockbuster film, and that the agency is exploring Mars for $\$ 300,000$ a day.

But, speaking at the Viking symposium, Noel Hinners warned that the agency's investment in technology was "totally inadequate" to support the ambitious missions planned for 2001 and after, particularly if the sample return is thrown in. Hinners is a former head of NASA's science office and now vice-president of flight systems at Lockheed Martin Corporation, which is building the 1996 and 1998 Surveyor spacecraft for NASA.

One possible way of affording a sample return while remaining within the Surveyor budget would be to scale down earlier missions in the series. But a recent analysis at JPL, which Shirley stresses was only preliminary, suggests that even this would not make up the shortfall. Bringing in international partners would be another possible solution. NASA is already talking to the Russians about a joint Mars mission in 2001.

NASA's human exploration office might also be able to contribute funds. But many technical and financial hurdles would still remain. One critical issue, says Shirley, will be arranging for quarantine of any samples returned from Mars, to avoid any risk of "back contamination".

Both the US missions heading for Mars this autumn will have substantial risks attached, largely as a result of their constrained budgets. The Mars Pathfinder, which is a technology test rather than a science mission, relies on an airbag system which has never previously been used to protect its lander on impact with the Martian surface. To save money, many of the on-board systems have no back-up.

The Mars Global Surveyor, which is due to be launched in December and duplicates much of the failed 1994 Mars Observer mission, will depend on a risky, months-long 'aerobraking' manoeuvre to decelerate in the Martian atmosphere. Russia's Mars 96 mission will also be launched in November, with an orbiter and two landers on board.

NASA's emphasis on economy bothers some engineers from the old school. Others are simply holding their breath. Louis Friedman, who follows Mars exploration as executive director of the Planetary Society, describes the forthcoming Mars 'Derby' as a "turning point" for the "cheaper, better, faster' approach. Tony Reichhardt

\section{Space voids really are devoid of matter}

London. A team of astronomers from Australia, Chile, Europe and the United States has discovered that the large empty regions in space, known as 'holes' or 'voids' (see right), are literally devoid of matter.

Astronomers have presumed for some time the existence in the Universe of 'dark matter', which cannot be seen but whose presence can be traced by its gravitational effect on visible matter, such as stars and galaxies. The newlydiscovered lack of dark matter within voids indicates that it is not smoothly distributed.

Astronomers from the European Southern Observatory (ESO) have now devised a technique that attempts to 'flush out' this 'invisible' matter, if there is any. Using optical and radio telescopes around the world, they measured the speeds of 2,000 well known galaxies. They then estimated the distribution of these galaxies on the basis of their observed velocities and positions.

Assuming interactions only through

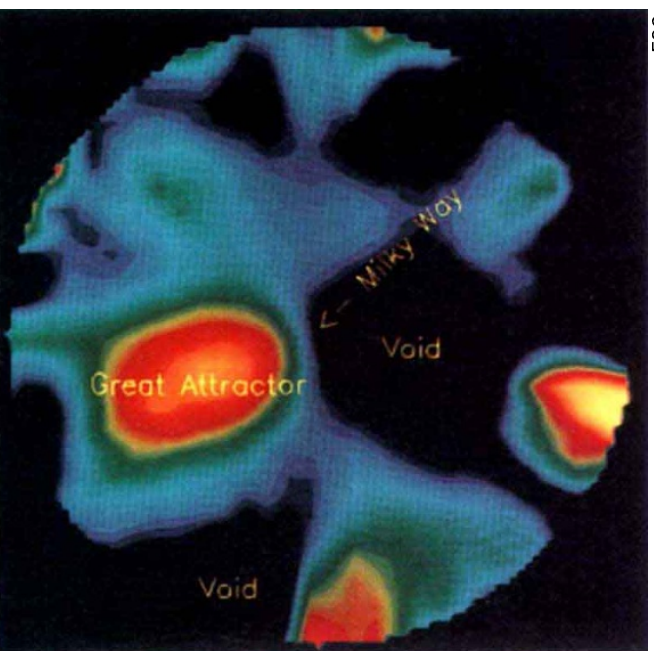

gravity, the astronomers estimated where the dark matter would have to be to produce the distribution and velocities of the real galaxies. To their surprise, they found several voids that do not need any matter at all.

The picture above is a simulation of these empty voids in the local Universe. The analysis will be published in the 1 September issue of Astrophysical Journal Letters. 